\title{
OSCILLATION AND NONOSCILLATION FOR IMPULSIVE DYNAMIC EQUATIONS ON CERTAIN TIME SCALES
}

\author{
MOUFFAK BENCHOHRA, SAMIRA HAMANI, AND JOHNNY HENDERSON
}

Received 1 December 2005; Revised 6 March 2006; Accepted 9 March 2006

We discuss the existence of oscillatory and nonoscillatory solutions for first-order impulsive dynamic equations on time scales with certain restrictions on the points of impulse. We will rely on the nonlinear alternative of Leray-Schauder type combined with a lower and upper solutions method.

Copyright (c) 2006 Mouffak Benchohra et al. This is an open access article distributed under the Creative Commons Attribution License, which permits unrestricted use, distribution, and reproduction in any medium, provided the original work is properly cited.

\section{Introduction}

This paper is concerned with the existence of oscillatory and nonoscillatory solutions of first-order impulsive dynamic equations on certain time scales. We consider the problem

$$
\begin{gathered}
y^{\Delta}(t)=f(t, y(t)), \quad t \in J_{\mathbb{T}}:=[0, \infty) \cap \mathbb{T}, t \neq t_{k}, k=1, \ldots, \\
y\left(t_{k}^{+}\right)=I_{k}\left(y\left(t_{k}^{-}\right)\right), \quad k=1, \ldots,
\end{gathered}
$$

where $\mathbb{T}$ is an unbounded-above time scale with $0 \in \mathbb{T} f: J_{\mathbb{T}} \times \mathbb{R} \rightarrow \mathbb{R}$ is a given function, $I_{k} \in C(\mathbb{R}, \mathbb{R}), t_{k} \in \mathbb{T}, 0=t_{0}<t_{1}<\cdots<t_{m}<t_{m+1}<\cdots<\infty, y\left(t_{k}^{+}\right)=\lim _{h \rightarrow 0^{+}} y\left(t_{k}+h\right)$ and $y\left(t_{k}^{-}\right)=\lim _{h \rightarrow 0^{+}} y\left(t_{k}-h\right)$ represent the right and left limits of $y(t)$ at $t=t_{k}$ in the sense of the time scale; that is, in terms of $h>0$ for which $t_{k}+h, t_{k}-h \in\left[t_{0}, \infty\right) \cap \mathbb{T}$, whereas if $t_{k}$ is left-scattered (resp., right-scattered), we interpret $y\left(t_{k}^{-}\right)=y\left(t_{k}\right)$ (resp., $\left.y\left(t_{k}^{+}\right)=y\left(t_{k}\right)\right)$.

Impulsive differential equations have become important in recent years in mathematical models of real processes and they rise in phenomena studied in physics, chemical technology, population dynamics, biotechnology and economics. There have been significant developments in impulse theory also in recent years, especially in the area of impulsive differential equations with fixed moments; see the monographs of Bainov and Simeonov [5], Lakshmikantham et al. [22], Samoilenko and Perestyuk [25], and the references therein. In recent years, dynamic equations on times scales have received much attention. 
We refer the reader to the books by Bohner and Peterson [10,11], Lakshmikantham et al. [23], and the references therein. The time scale calculus has tremendous potential for applications in mathematical models of real processes, for example, in physics, chemical technology, population dynamics, biotechnology and economics, neural networks, social sciences; see the monographs of Aulbach and Hilger [4], Bohner and Peterson [10, 11], Lakshmikantham et al. [23], and the references therein. The existence of solutions of boundary value problem on a measure chain (i.e., time scale) was recently studied by Henderson [20] and Henderson and Tisdell [21]. The question of existence of solutions to some classes of impulsive dynamic equations on time scales was treated very recently by Henderson [19] and Benchohra et al. in $[1,7,8]$. The aim of this paper is to initiate the study of oscillatory and nonoscillatory solutions to impulsive dynamic equations on time scales. For oscillation and nonoscillation of impulsive differential equations, see, for instance, the monograph of Bainov and Simonov [5] and the papers of Graef et al. [16, 17]. The purpose of this paper is to give some sufficient conditions for existence of oscillatory and nonoscillatory solutions of the first-order dynamic impulsive problem (1.1) on time scales. There has been, in fact, a good deal of research already devoted to oscillation questions for dynamic equations on time scales; see, for example, $[2,9,12,14,15,24]$. For the purposes of this paper, we will rely on the nonlinear alternative of Leray-Schauder type combined with a lower and upper solutions method. Our results can be considered as contributions to this emerging field.

\section{Preliminaries}

We will briefly recall some basic definitions and facts from time scale calculus that we will use in the sequel.

A time scale $\mathbb{T}$ is an closed subset of $\mathbb{R}$. It follows that the jump operators $\sigma, \rho: \mathbb{T} \rightarrow \mathbb{T}$ defined by

$$
\sigma(t)=\inf \{s \in \mathbb{T}: s>t\}, \quad \rho(t)=\sup \{s \in \mathbb{T}: s<t\}
$$

(supplemented by $\inf \varnothing:=\sup \mathbb{T}$ and $\sup \varnothing:=\inf \mathbb{\mathbb { T }}$ ) are well defined. The point $t \in \mathbb{T}$ is left-dense, left-scattered, right-dense, right-scattered if $\rho(t)=t, \rho(t)<t, \sigma(t)=t, \sigma(t)\rangle$ $t$, respectively. If $\mathbb{T}$ has a right-scattered minimum $m$, define $\mathbb{T}_{k}:=\mathbb{T}-\{m\}$; otherwise, set $\mathbb{T}_{k}=\mathbb{T}$. If $\mathbb{T}$ has a left-scattered maximum $M$, define $\mathbb{T}^{k}:=\mathbb{T}-\{M\}$; otherwise, set $\mathbb{T}^{k}=\mathbb{T}$. The notations $[a, b],[a, b)$, and so on will denote time scales intervals

$$
[a, b]=\{t \in \mathbb{T}: a \leq t \leq b\},
$$

where $a, b \in \mathbb{T}$ with $a<\rho(b)$.

Definition 2.1. Let $X$ be a Banach space. The function $g: \mathbb{T} \rightarrow X$ will be called $r d-$ continuous provided it is continuous at each right-dense point and has a left-sided limit at each point, and write $g \in C_{r d}(\mathbb{T})=C_{r d}(\mathbb{T}, X)$. For $t \in \mathbb{T}^{k}$, the $\Delta$ derivative of $g$ at $t$, denoted by $g^{\Delta}(t)$, is the number (provided it exists) such that for all $\varepsilon>0$, there exists a neighborhood $U$ of $t$ such that

$$
\left|g(\sigma(t))-g(s)-g^{\Delta}(t)[\sigma(t)-s]\right| \leq \varepsilon|\sigma(t)-s|
$$


for all $s \in U$. A function $F$ is called an antiderivative of $g: \mathbb{T} \rightarrow X$ provided

$$
F^{\Delta}(t)=g(t) \quad \text { for each } t \in \mathbb{T}^{k}
$$

A function $g: \mathbb{T} \rightarrow \mathbb{R}$ is called regressive if

$$
1+\mu(t) g(t) \neq 0 \quad \forall t \in \mathbb{T},
$$

where $\mu(t)=\sigma(t)-t$ which is called the graininess function. The set of all $r d$-continuous functions $g$ that satisfy $1+\mu(t) g(t)>0$ for all $t \in \mathbb{T}$ will be denoted by $\mathscr{R}^{+}$.

The generalized exponential function $e_{p}$ is defined as the unique solution $y(t)=$ $e_{p}(t, a)$ of the initial value problem $y^{\Delta}=p(t) y, y(a)=1$, where $p$ is a regressive function. An explicit formula for $e_{p}(t, a)$ is given by

$$
e_{p}(t, s)=\exp \left\{\int_{s}^{t} \xi_{\mu(\tau)}(p(\tau)) \Delta \tau\right\} \quad \text { with } \xi_{h}(z)= \begin{cases}\frac{\log (1+h z)}{h} & \text { if } h \neq 0 \\ z & \text { if } h=0\end{cases}
$$

For more details, see [10]. Clearly, $e_{p}(t, s)$ never vanishes. $C([0, b], \mathbb{R})$ is the Banach space of all continuous functions from $[a, b]$ into $\mathbb{R}$, where $[a, b] \subset \mathbb{T}$ with the norm

$$
\|y\|_{\infty}=\sup \{|y(t)|: t \in[a, b]\}
$$

Remark 2.2. (i) If $f$ is continuous, then $f r d$-continuous.

(ii) If $f$ is delta differentiable at $t$, then $f$ is continuous at $t$.

\section{Main result}

We will assume for the remainder of the paper that, for each $k=1, \ldots$, the points of impulse $t_{k}$ are right-dense. In order to define the solution of (1.1), we will consider the space

$$
\begin{gathered}
P C=\left\{y: J_{\mathbb{T}} \longrightarrow \mathbb{R}: y_{k} \in C\left(J_{k}, \mathbb{R}\right), k=0,1, \ldots,\right. \text { and there exist } \\
\left.y\left(t_{k}^{-}\right) \text {and } y\left(t_{k}^{+}\right), k=1, \ldots, \text { with } y\left(t_{k}^{-}\right)=y\left(t_{k}\right)\right\},
\end{gathered}
$$

where $y_{k}$ is the restriction of $y$ to $J_{k}=\left[t_{k}, t_{k+1}\right]$.

Remark 3.1. In light of the right-density assumption on each impulse point, we observe that this restriction precludes certain time scales. For example, time scales that are excluded from this work include discrete time scales, time scales associated with $q$-differences, harmonic numbers time scales, and so forth. We observe further that, in the context of impulsive problems on time scales, such restrictions on impulse points are not uncommon; see, for example, $[13,19]$. 
Let us start by defining what we mean by a solution of problem (1.1).

Definition 3.2. A function $y \in P C \cap C^{1}\left(\left(t_{k}, t_{k+1}\right), \mathbb{R}\right), k=0, \ldots$, is said to be a solution of (1.1) if $y$ satisfies the equation $y^{\Delta}(t)=f(t, y(t))$ on $J \backslash\left\{t_{1}, \ldots\right\}$ and the condition $y\left(t_{k}^{+}\right)=$ $I_{k}\left(y\left(t_{k}^{-}\right)\right), k=1, \ldots$.

Definition 3.3. A function $\alpha \in P C \cap C^{1}\left(\left(t_{k}, t_{k+1}\right), \mathbb{R}\right), k=0, \ldots$, is said to be a lower solution of $(1.1)$ if $\alpha^{\Delta}(t) \leq f(t, \alpha(t))$ on $J_{\mathbb{T}} \backslash\left\{t_{1}, \ldots\right\}$ and $\alpha\left(t_{k}^{+}\right) \leq I_{k}\left(\alpha\left(t_{k}\right)\right), k=1, \ldots$. Similarly, a function $\beta \in P C \cap C^{1}\left(\left(t_{k}, t_{k+1}\right), \mathbb{R}\right), k=0, \ldots$, is said to be an upper solution of (1.1) if $\beta^{\Delta}(t) \geq f(t, \beta(t))$ on $J_{\mathbb{T}} \backslash\left\{t_{1}, \ldots\right\}$ and $\beta\left(t_{k}^{+}\right) \geq I_{k}\left(\beta\left(t_{k}\right)\right), k=1, \ldots$

For the study of this problem, we first list the following hypotheses:

(H1) the function $f: J_{\mathbb{T}} \times \mathbb{R} \rightarrow \mathbb{R}$ is continuous;

(H2) for all $r>0$, there exists a nonnegative function $h_{r} \in C\left(J_{\mathbb{T}}, \mathbb{R}^{+}\right)$with

$$
|f(t, y)| \leq h_{r}(t) \quad \forall t \in J_{\mathbb{T}} \text { and all }|y| \leq r ;
$$

(H3) there exist $\alpha$ and $\beta \in P C \cap C^{1}\left(\left(t_{k}, t_{k+1}\right), \mathbb{R}\right), k=0, \ldots$, lower and upper solutions for the problem (1.1) such that $\alpha \leq \beta$;

$$
\alpha\left(t_{k}^{+}\right) \leq \min _{y \in\left[\alpha\left(t_{k}^{-}\right), \beta\left(t_{k}^{-}\right)\right]} I_{k}(y) \leq \max _{y \in\left[\alpha\left(t_{k}^{-}\right), \beta\left(t_{k}^{-}\right)\right]} I_{k}(y) \leq \beta\left(t_{k}^{+}\right), \quad k=1, \ldots
$$

Theorem 3.4. Assume that hypotheses (H1)-(H4) hold. Then the problem (1.1) has at least one solution $y$ such that

$$
\alpha(t) \leq y(t) \leq \beta(t) \quad \forall t \in J
$$

Proof. The proof will be given in several steps.

Step 1. Consider the problem

$$
y^{\Delta}(t)=f(t, y(t)), \quad t \in J_{1}:=\left[t_{0}, t_{1}\right] .
$$

Transform the problem (3.5) into a fixed point problem. Consider the following modified problem:

$$
y^{\Delta}(t)+y(t)=f_{1}(t, y(t)), \quad t \in J_{1},
$$

where

$$
\begin{gathered}
f_{1}(t, y)=f(t, \tau(t, y))+\tau(t, y), \\
\tau(t, y)=\max \{\alpha(t), \min (y, \beta(t))\}, \\
\bar{y}(t)=\tau(t, y) .
\end{gathered}
$$

A solution to (3.6) is a fixed point of the operator $N: C\left(\left[t_{0}, t_{1}\right], \mathbb{R}\right) \rightarrow C\left(\left[t_{0}, t_{1}\right], \mathbb{R}\right)$ defined by

$$
N(y)(t)=\int_{t_{0}}^{t}\left[f_{1}(s, \bar{y}(s))+\bar{y}(s)-y(s)\right] \Delta s .
$$


Remark 3.5. (i) Notice that $f_{1}$ is a continuous function, and from (H2) there exists $M^{*}>0$ such that

$$
\left\|f_{1}(t, y)\right\| \leq M^{*}+\max \left\{\sup _{t \in J_{1}}|\alpha(t)| \sup _{t \in J_{1}}|\beta(t)|\right\}:=M .
$$

(ii) By the definition of $\tau$ it is clear that

$$
\alpha\left(t_{k}^{+}\right) \leq I_{k}\left(\tau\left(t_{k}, y\left(t_{k}\right)\right)\right) \leq \beta\left(t_{k}^{+}\right), \quad k=1, \ldots
$$

In order to apply the nonlinear alternative of Leray-Schauder type, we first show that $\mathrm{N}$ is continuous and completely continuous.

Claim 1. $N$ is continuous.

Let $\left\{y_{n}\right\}$ be a sequence such that $y_{n} \rightarrow y$ in $C\left(\left[t_{0}, t_{1}\right], \mathbb{R}\right)$. Then

$$
\begin{aligned}
\left|N\left(y_{n}\right)(t)-N(y)(t)\right| \leq & \int_{t_{0}}^{t}\left[\left|f_{1}\left(s, \bar{y}_{n}(s)\right)-f_{1}(s, \bar{y}(s)) \Delta s\right|\right. \\
& \left.\quad+\left|\bar{y}_{n}(s)-\bar{y}(s)\right|+\left|y_{n}(s)-y(s)\right|\right] \Delta s \\
\leq & \int_{t_{0}}^{t}\left|f_{1}\left(s, \bar{y}_{n}(s)\right)-f_{1}(s, \bar{y}(s)) \Delta s\right| \\
& +\left(t_{1}-t_{0}\right)\left\|\bar{y}_{n}(s)-\bar{y}(s)\right\|_{\infty}+\left(t_{1}-t_{0}\right)\left\|y_{n}(s)-y(s)\right\|_{\infty} \Delta s .
\end{aligned}
$$

Since $f_{1}$ is a continuous function, then we have

$$
\left\|N\left(y_{n}\right)-N(y)\right\|_{\infty} \leq\left\|f_{1}\left(\cdot, \bar{y}_{n}\right)-f_{1}(\cdot, \bar{y})\right\|_{\infty}+\left(t_{1}-t_{0}\right)\left\|\bar{y}_{n}-\bar{y}\right\|_{\infty}+\left(t_{1}-t_{0}\right)\left\|y_{n}-y\right\|_{\infty} .
$$

Thus

$$
\left\|N\left(y_{n}\right)-N(y)\right\|_{\infty} \longrightarrow 0 \quad \text { as } n \longrightarrow \infty
$$

Claim 2. $N$ maps bounded sets into bounded sets in $C\left(\left[t_{0}, t_{1}\right], \mathbb{R}\right)$.

Indeed, it is enough to show that there exists a positive constant $\ell$ such that for each $y \in B_{q}=\left\{y \in C\left(\left[t_{0}, t_{1}\right], \mathbb{R}\right):\|y\|_{\infty} \leq q\right\}$ one has $\|N y\|_{\infty} \leq \ell$. Let $y \in B_{q}$. Then for each $t \in J_{1}$ we have

$$
N(y)(t)=\int_{t_{0}}^{t}\left[f_{1}(s, \bar{y}(s))+\bar{y}(s)-y(s)\right] \Delta s .
$$


By (H1) and Remark 3.5 we have, for each $t \in J_{1}$,

$$
\begin{aligned}
|N y(t)| \leq & \int_{t_{0}}^{t}\left[\left|f_{1}(t, y)\right|+|\bar{y}(s)|+|y(s)|\right] \Delta s+ \\
\leq & \left(t-t_{0}\right) M+\left(t-t_{0}\right) \max \left\{q, \sup _{t \in J_{1}}|\alpha(t)|, \sup _{t \in J_{1}}|\beta(t)|\right\} \\
& +\left(t-t_{0}\right) q:=\ell .
\end{aligned}
$$

Thus $\|N(y)\|_{\infty} \leq \ell$.

Claim 3. $N$ maps bounded set into equicontinuous sets of $P C$.

Let $u_{1}, u_{2} \in J_{1}, u_{1}<u_{2}$ and $B_{q}$ be a bounded set of $P C$ as in Claim 2. Let $y \in B_{q}$. Then

$$
\begin{aligned}
& \left|N\left(u_{2}\right)-N\left(u_{1}\right)\right| \\
& \quad \leq\left(u_{2}-u_{1}\right) M+\left(u_{2}-u_{1}\right) \max \left\{q, \sup _{t \in J_{1}}|\alpha(t)|, \sup _{t \in J_{1}}|\beta(t)|\right\}+\left(u_{2}-u_{1}\right) q .
\end{aligned}
$$

As $u_{2} \rightarrow u_{1}$, the right-hand side of the above inequality tends to zero.

As a consequence of Claims 1 to 3 together with the Arzela-Ascoli theorem, we can conclude that $N: C\left(\left[t_{0}, t_{1}\right], \mathbb{R}\right) \rightarrow C\left(\left[t_{0}, t_{1}\right], \mathbb{R}\right)$ is continuous and completely continuous.

Claim 4. A priori bounds on solutions.

Let $y$ be a possible solution of $y=\lambda N(y)$ with $\lambda \in[0,1]$. Then we have

$$
y(t)=\lambda \int_{t_{0}}^{t}\left[f_{1}(t, y)+\bar{y}(s)-y(s)\right] \Delta s
$$

This implies by Remark 3.5 that for each $t \in J_{1}$ we have

$$
\begin{aligned}
|y(t)| & =\left|\int_{t_{0}}^{t}\left[f_{1}(t, y)+\bar{y}(s)+y(s)\right] \Delta s\right| \leq \int_{t_{0}}^{t}\left[\left|f_{1}(t, y)\right|+|\bar{y}(s)|+|y(s)|\right] \Delta s \\
& \leq\left(t-t_{0}\right) M+\left(t-t_{0}\right) \max \left(\sup _{t \in J_{1}}|\alpha(t)|, \sup _{t \in J_{1}}|\beta(t)|\right)+\int_{t_{0}}^{t}|y(s)| \Delta s .
\end{aligned}
$$

Now $1 \in \mathscr{R}^{+}$. Hence, let $e_{1}(t, 0)$ be the unique solution of the problem

$$
y^{\Delta}(t)=y(t), \quad y(0)=1 .
$$

Then from Gronwall's inequality we have

$$
|y(t)| \leq f_{*}+f_{*} \int_{t_{0}}^{t} e_{1}(t, \sigma(s)) \Delta s,
$$


where

$$
f_{*}=M\left(t-t_{0}\right)+\left(t-t_{0}\right) \max \left\{\sup _{t \in J_{1}}|\alpha(t)|, \sup _{t \in J_{1}}|\beta(t)|\right\} \text {. }
$$

Thus

$$
\|y\|_{\infty} \leq f_{*}+f_{*} \sup _{t \in J_{1}} \int_{t_{0}}^{t} e_{1}(t, \sigma(s)) \Delta s:=M_{1} .
$$

Set

$$
U=\left\{y \in C\left(\left[t_{0}, t_{1}\right], \mathbb{R}\right):\|y\|_{\infty}<M_{1}+1\right\} .
$$

From the choice of $U$ there is no $y \in \partial U$ such that $y=\lambda N(y)$ for some $\lambda \in(0,1)$. As a consequence of the nonlinear alternative of Leray-Schauder type [18], we deduce that $N$ has a fixed point $y$ in $U$ which is a solution of the problem (3.6).

Claim 5. The solution $y$ of (3.6) satisfies

$$
\alpha(t) \leq y(t) \leq \beta(t) \quad \forall t \in J_{1} .
$$

Let $y$ be the above solution to (3.6). We prove that

$$
\alpha(t) \leq y(t) \quad \forall t \in J_{1}
$$

Suppose not. Then there exist $e_{1}, e_{2} \in J_{1}, e_{1}<e_{2}$ such that $\alpha\left(e_{1}\right)=y\left(e_{1}\right)$ and

$$
y(t)<\alpha(t) \quad \forall t \in\left(e_{1}, e_{2}\right] .
$$

In view of the definition of $\tau$ one has

$$
y(t)-y\left(e_{1}\right)=\int_{e_{1}}^{t}[f(s, \alpha(s))-(y(s)-\alpha(s))] \Delta s .
$$

Using the fact that $\alpha$ is a lower solution to (3.6), the above inequality yields

$$
\begin{aligned}
\alpha(t)-\alpha\left(e_{1}\right) & \leq \int_{e_{1}}^{t} f(s, \alpha(s)) \Delta s<\int_{e_{1}}^{t}[f(s, \alpha(s))-(y(s)-\alpha(s))] \Delta s \\
& =y(t)-y\left(e_{1}\right)<\alpha(t)-\alpha\left(e_{1}\right),
\end{aligned}
$$

which is a contradiction. Analogously, we can prove that

$$
y(t) \leq \beta(t) \quad \forall t \in\left[t_{0}, t_{1}\right] .
$$

This shows that the problem (3.6) has a solution in the interval $[\alpha, \beta]$ which is solution of (3.5). Denote this solution by $y_{0}$. 
Step 2. Consider the following problem:

$$
\begin{gathered}
y^{\Delta}(t)=f(t, y(t)), \quad t \in J_{2}:=\left[t_{1}, t_{2}\right], \\
y\left(t_{1}^{+}\right)=I_{1}\left(y_{0}\left(t_{1}^{-}\right)\right) .
\end{gathered}
$$

Consider the following modified problem:

$$
\begin{gathered}
y^{\Delta}(t)+y(t)=f_{1}(t, y(t)), \quad t \in J_{2}, \\
y\left(t_{1}^{+}\right)=I_{1}\left(y_{0}\left(t_{1}^{-}\right)\right) .
\end{gathered}
$$

A solution to (3.31)-(3.32) is a fixed point of the operator $N_{1}: C\left(\left[t_{1}, t_{2}\right], \mathbb{R}\right) \rightarrow C\left(\left[t_{1}, t_{2}\right]\right.$, $\mathbb{R})$ defined by

$$
N_{1}(y)(t)=\int_{t_{1}}^{t}\left[f_{1}(s, \bar{y}(s))+\bar{y}(s)-y(s)\right] \Delta s+I_{1}\left(y_{0}\left(t_{1}^{-}\right)\right)
$$

Since $y_{0}\left(t_{1}\right) \in\left[\alpha\left(t_{1}^{-}\right), \beta\left(t_{1}^{-}\right)\right]$, then (H4) implies that

$$
\alpha\left(t_{1}^{+}\right) \leq I_{1}\left(y_{0}\left(t_{1}^{-}\right)\right) \leq \beta\left(t_{1}^{+}\right),
$$

that is,

$$
\alpha\left(t_{1}^{+}\right) \leq y\left(t_{1}^{+}\right) \leq \beta\left(t_{1}^{+}\right)
$$

Using the same reasoning as that used for problem (3.5), we can conclude the existence of at least one solution $y$ to (3.32)-(3.41). We now show that this solution satisfies

$$
\alpha(t) \leq y(t) \leq \beta(t) \quad \forall t \in J_{2} .
$$

Let $y$ be the above solution to (3.32)-(3.41). We show that

$$
\alpha(t) \leq y(t) \quad \forall t \in J_{2}
$$

Assume this is false. Then since $y\left(t_{1}^{+}\right) \geq \alpha\left(t_{1}^{+}\right)$, there exist $e_{3}, e_{4} \in J_{2}$ with $e_{3}<e_{4}$ such that $\alpha\left(e_{3}\right)=y\left(e_{3}\right)$ and

$$
y(t)<\alpha(t) \quad \forall t \in\left(e_{1}, e_{2}\right]
$$

In view of the definition of $\tau$ one has

$$
\begin{aligned}
\alpha(t)-\alpha\left(e_{3}\right) & \leq \int_{e_{3}}^{t} f(s, \alpha(s)) \Delta s<\int_{e_{3}}^{t}[f(s, \alpha(s))-(y(s)-\alpha(s))] \Delta s \\
& =y(t)-y\left(e_{3}\right)<\alpha(t)-\alpha\left(e_{3}\right),
\end{aligned}
$$

which is a contradiction. Analogously, we can prove that

$$
y(t) \leq \beta(t) \quad \forall t \in\left[t_{1}, t_{2}\right] .
$$


This shows that the problem (3.32)-(3.41) has a solution in the interval $[\alpha, \beta]$ which is a solution of (3.30). Denote this solution by $y_{1}$.

Step 3. We continue this process and take into account that $y_{m}:=\left.y\right|_{\left[t_{m-1}, t_{m}\right]}$ is a solution to the problem

$$
\begin{gathered}
y^{\Delta}(t)=f(t, y(t)), \quad t \in J_{m}:=\left[t_{m-1}, t_{m}\right], \\
y\left(t_{m}^{+}\right)=I_{m}\left(y_{m-1}\left(t_{m-1}^{-}\right)\right) .
\end{gathered}
$$

Consider the following modified problem:

$$
\begin{gathered}
y^{\Delta}(t)+y(t)=f_{1}(t, y(t)), \quad t \in J_{m}, \\
y\left(t_{m}^{+}\right)=I_{m}\left(y_{m-1}\left(t_{m-1}^{-}\right)\right) .
\end{gathered}
$$

A solution to $(3.43)$ is a fixed point of the operator $N_{m}: C\left(\left[t_{m-1}, t_{m}\right], \mathbb{R}\right) \rightarrow C\left(\left[t_{m-1}, t_{m}\right], \mathbb{R}\right)$ defined by

$$
N_{m}(y)(t)=\int_{t_{m}}^{t}\left[f_{1}(s, \bar{y}(s))+\bar{y}(s)-y(s)\right] \Delta s+I_{m}\left(y\left(t_{m-1}^{-}\right)\right) .
$$

Using the same reasoning as that used for problems (3.5) and (3.6)-(3.30), we can conclude the existence of at least one solution $y$ to (3.41)-(3.42). Denote this solution by $y_{m-1}$.

The solution $y$ of the problem (1.1) is then defined by

$$
y(t)= \begin{cases}y_{0}(t), & t \in\left[t_{0}, t_{1}\right], \\ y_{2}(t), & t \in\left(t_{1}, t_{2}\right], \\ \vdots & \\ y_{m-1}(t), & t \in\left(t_{m-1}, t_{m}\right], \\ \vdots & \end{cases}
$$

The proof is complete.

The following theorem gives sufficient conditions to ensure the nonoscillation of solutions of problem (1.1).

Theorem 3.6. Let $\alpha$ and $\beta$ be lower and upper solutions, respectively, of (1.1) with $\alpha \leq \beta$ and assume that

(H5) $\alpha$ is eventually positive nondecreasing, or $\beta$ is eventually negative nonincreasing. Then every solution $y$ of (1.1) such that $y \in[\alpha, \beta]$ is nonoscillatory.

Proof. Assume $\alpha$ to be eventually positive. Thus there exists $T_{\alpha}>t_{0}$ such that

$$
\alpha(t)>0 \quad \forall t>T_{\alpha} .
$$


Hence, $y(t)>0$ for all $t>T_{\alpha}$, and $t \neq t_{k}, k=1, \ldots$. For some $k \in \mathbb{N}$ and $t>t_{\alpha}$, we have $y\left(t_{k}^{+}\right)=I_{k}\left(y\left(t_{k}\right)\right)$. From (H4) we get $y\left(t_{k}^{+}\right)>\alpha\left(t_{k}^{+}\right)$. Since for each $h>0, \alpha\left(t_{k}+h\right) \geq \alpha\left(t_{k}\right)>$ 0 , then $I_{k}\left(y\left(t_{k}\right)\right)>0$ for all $t_{k}>T_{\alpha}, k=1, \ldots$, which means that $y$ is nonoscillatory. Analogously, if $\beta$ is eventually negative, then there exists $T_{\beta}>t_{0}$ such that

$$
y(t)<0 \quad \forall t>T_{\beta},
$$

which means that $y$ is nonoscillatory. This completes the proof.

The following theorem discusses the oscillation of solutions of problem (1.1).

Theorem 3.7. Let $\alpha$ and $\beta$ be lower and upper solutions, respectively, of (1.1), and assume that the sequences $\alpha\left(t_{k}\right)$ and $\beta\left(t_{k}\right), k=1, \ldots$, are oscillatory. Then every solution $y$ of (1.1) such that $y \in[\alpha, \beta]$ is oscillatory.

Proof. Suppose on the contrary that $y$ is a nonoscillatory solution of (1.1). Then there exists $T_{y}>0$ such that $y(t)>0$ for all $t>T_{y}$, or $y(t)<0$ for all $t>T_{y}$. In the case $y(t)>0$ for all $t>T_{y}$, we have $\beta\left(t_{k}\right)>0$ for all $t_{k}>T_{y}, k=1, \ldots$, which is a contradiction, since $\beta\left(t_{k}\right)$ is an oscillatory upper solution. Analogously, in the case $y(t)<0$ for all $t>T_{y}$, we have $\alpha\left(t_{k}\right)<0$ for all $t_{k}>T_{y}, k=1, \ldots$, which is also a contradiction, since $\alpha\left(t_{k}\right)$ is an oscillatory lower solution.

\section{An example}

As an application of our results, we consider the following impulsive dynamic equation

$$
\begin{gathered}
y^{\Delta}(t)=f(t, y), \quad \text { for each } t \in J_{\mathbb{T}}:=[0, \infty) \cap \mathbb{T}, t \neq t_{k}, k=1, \ldots, \\
y\left(t_{k}^{+}\right)=I_{k}\left(y\left(t_{k}^{-}\right)\right), \quad k \in \mathbb{N},
\end{gathered}
$$

where $f: J_{\mathbb{I}} \times \mathbb{R} \rightarrow \mathbb{R}$. Assume that there exist $g_{1}(\cdot), g_{2}(\cdot) \in C\left(J_{\mathbb{T}}, \mathbb{R}\right)$ such that

$$
g_{1}(t) \leq f(t, y) \leq g_{2}(t) \quad \forall t \in J_{\mathbb{T}}, y \in \mathbb{R}
$$

and, for each $t \in J_{\mathbb{T}}$,

$$
\begin{array}{ll}
\int_{0}^{t} g_{1}(s) \Delta s \leq I_{k}\left(\int_{0}^{t} g_{1}(s) \Delta s\right), & k \in \mathbb{N} \\
\int_{0}^{t} g_{2}(s) \Delta s \geq I_{k}\left(\int_{0}^{t} g_{2}(s) \Delta s\right), & k \in \mathbb{N} .
\end{array}
$$

Consider the functions $\alpha(t):=\int_{0}^{t} g_{1}(s) \Delta s$ and $\beta(t):=\int_{0}^{t} g_{2}(s) \Delta s$. Clearly, $\alpha$ and $\beta$ are lower and upper solutions of the problem (4.1), respectively; that is,

$$
\begin{aligned}
& \alpha^{\Delta}(t) \leq f(t, y) \quad \forall t \in J_{\mathbb{T}} \quad \text { and all } y \in \mathbb{R}, \\
& \beta^{\Delta}(t) \geq f(t, y) \quad \forall t \in J_{\mathbb{T}} \quad \text { and all } y \in \mathbb{R} \text {. }
\end{aligned}
$$


Since all the conditions of Theorem 3.4 are satisfied, the problem (4.1) has at least one solution $y$ on $J_{\mathbb{U}}$ with $\alpha \leq y \leq \beta$. If $g_{1}(t)>0$, then $\alpha$ is positive and nondecreasing, thus $y(t)$ is nonoscillatory. If $g_{2}(t)<0$, then $\beta$ is negative and nonincreasing, thus $y(t)$ is nonoscillatory. If the sequences $\alpha\left(t_{k}\right)$ and $\beta\left(t_{k}\right)$ are both oscillatory, then $y(t)$ is oscillatory.

\section{Acknowledgments}

The authors are grateful for the detailed reports and suggestions offered by each referee. These have led to improvements in the presentation of the paper's results.

\section{References}

[1] R. P. Agarwal, M. Benchohra, D. O’Regan, and A. Ouahab, Second order impulsive dynamic equations on time scales, Functional Differential Equations 11 (2004), no. 3-4, 223-234.

[2] R. P. Agarwal, M. Bohner, and S. H. Saker, Oscillation of second order delay dynamic equations, to appear in The Canadian Applied Mathematics Quarterly.

[3] R. P. Agarwal, S. R. Grace, and D. O'Regan, Oscillation Theory for Second Order Dynamic Equations, Series in Mathematical Analysis and Applications, vol. 5, Taylor \& Francis, London, 2003.

[4] B. Aulbach and S. Hilger, Linear dynamic processes with inhomogeneous time scale, Nonlinear Dynamics and Quantum Dynamical Systems (Gaussig, 1990), Math. Res., vol. 59, Akademie, Berlin, 1990, pp. 9-20.

[5] D. D. Bainov and P. S. Simeonov, Systems with Impulse Effect, Ellis Horwood Series: Mathematics and Its Applications, Ellis Horwood, Chichester, 1989.

[6] _ Oscillation Theory of Impulsive Differential Equations, International Publications, Florida, 1998.

[7] M. Benchohra, J. Henderson, S. K. Ntouyas, and A. Ouahab, On first order impulsive dynamic equations on time scales, Journal of Difference Equations and Applications 10 (2004), no. 6, 541548.

[8] M. Benchohra, S. K. Ntouyas, and A. Ouahab, Existence results for second order boundary value problem of impulsive dynamic equations on time scales, Journal of Mathematical Analysis and Applications 296 (2004), no. 1, 65-73.

[9] E. A. Bohner, M. Bohner, and S. H. Saker, Oscillation criteria for a certain class of second order Emden-Fowler dynamic equations, to appear in Electronic Transactions on Numerical Analysis.

[10] M. Bohner and A. Peterson, Dynamic Equations on Time Scales. An Introduction with Applications, Birkhäuser Boston, Massachusetts, 2001.

[11] M. Bohner and A. Peterson (eds.), Advances in Dynamic Equations on Time Scales, Birkhäuser Boston, Massachusetts, 2003.

[12] M. Bohner and S. H. Saker, Oscillation of second order nonlinear dynamic equations on time scales, The Rocky Mountain Journal of Mathematics 34 (2004), no. 4, 1239-1254.

[13] Y. K. Chang and W. T. Li, Existence results for impulsive dynamic equations on time scales with nonlocal initial conditions, Mathematical and Computer Modelling 43 (2006), no. 3-4, 377-384.

[14] L. Erbe, Oscillation criteria for second order linear equations on a time scale, The Canadian Applied Mathematics Quarterly 9 (2001), no. 4, 345-375 (2002).

[15] L. Erbe, A. Peterson, and S. H. Saker, Oscillation criteria for second-order nonlinear dynamic equations on time scales, Journal of the London Mathematical Society. Second Series 67 (2003), no. 3 , 701-714.

[16] J. R. Graef and J. Karsai, On the oscillation of impulsively damped halflinear oscillators, Proceedings of the 6th Colloquium on the Qualitative Theory of Differential Equations (Szeged, 1999), Proc. Colloq. Qual. Theory Differ. Equ., no. 14, Electron. J. Qual. Theory Differ. Equ., Szeged, 2000, p. 12. 
[17] __ Oscillation and nonoscillation in nonlinear implusive system with increasing energy, Discrete and Continuous Dynamical Systems 7 (2000), 161-173, Proceeding of the 3rd International Conference on Dynamical systems and Differential Equations.

[18] A. Granas and J. Dugundji, Fixed Point Theory, Springer Monographs in Mathematics, Springer, New York, 2003.

[19] J. Henderson, Double solutions of impulsive dynamic boundary value problems on a time scale, Journal of Difference Equations and Applications 8 (2002), no. 4, 345-356.

[20] _ Nontrivial solutions to a nonlinear boundary value problem on a time scale, Communications on Applied Nonlinear Analysis 11 (2004), no. 1, 65-71.

[21] J. Henderson and C. C. Tisdell, Topological transversality and boundary value problems on time scales, Journal of Mathematical Analysis and Applications 289 (2004), no. 1, 110-125.

[22] V. Lakshmikantham, D. D. Bainov, and P. S. Simeonov, Theory of Impulsive Differential Equations, Series in Modern Applied Mathematics, vol. 6, World Scientific, New Jersey, 1989.

[23] V. Lakshmikantham, S. Sivasundaram, and B. Kaymakcalan, Dynamic Systems on Measure Chains, Mathematics and Its Applications, vol. 370, Kluwer Academic, Dordrecht, 1996.

[24] S. H. Saker, Oscillation of nonlinear dynamic equations on time scales, Applied Mathematics and Computation 148 (2004), no. 1, 81-91.

[25] A. M. Samoilenko and N. A. Perestyuk, Impulsive Differential Equations, World Scientific Series on Nonlinear Science. Series A: Monographs and Treatises, vol. 14, World Scientific, New Jersey, 1995.

Mouffak Benchohra: Laboratoire de Mathématiques, Université de Sidi Bel Abbès, BP 89, 22000 Sidi Bel Abbès, Algeria

E-mail address: benchohra@yahoo.com

Samira Hamani: Laboratoire de Mathématiques, Université de Sidi Bel Abbès, BP 89, 22000 Sidi Bel Abbès, Algeria

E-mail address: hamani_samira@yahoo.fr

Johnny Henderson: Department of Mathematics, Baylor University, Waco, Tx 76798-7328, USA

E-mail address: johnny_henderson@baylor.edu 